\title{
PLEOMORPHIC ADENOMA OF PALATE
}

\author{
Fernando $\mathbf{R}^{1}$, Bandara D M S M ${ }^{2}$, Perera W A T M N² \\ ${ }^{1}$ Professor in Surgery, ${ }^{2}$ Senior Registrar, University Surgical Unit, \\ North Colombo Teaching Hospital, Ragama.
}

R Fernando MS, FRCS

Professor in Surgery, University Surgical Unit, North Colombo Teaching Hospital, Ragama, Sri Lanka.

D M S M Bandara MD (Surgery), MRCS

Senior Registrar, University Surgical Unit, North Colombo Teaching Hospital, Ragama, Sri Lanka.

WA T M N Perera MD (Surgery), MRCS

Senior Registrar, University Surgical Unit, North Colombo Teaching Hospital, Ragama, Sri Lanka.

Corresponding author:

R Fernando, 8/3 Ragama Road, Kadawatha

Tel: 0112901342

E-mail: ranilfern@sltnet.lk

58-year old man presented with slow growing painless lump in the centre of hard palate three months duration. He had tenderness, bleeding from mouth or nose, difficulty in swallowing or breathing. Examination revealed a non tender spherical lump in the centre of inferior surface of the posterior hard palate (Figure 1). Fine needle aspiration cytology revealed it to be pleomorphic adenoma. Upper gastro intestinal endoscopy was normal. Examination under anaesthesia showed no other lesion. Wide local excision was performed. Histology was compatible with benign pleomorphic adenoma of palate.

Pleomorphic adenoma contains elements of both epithelial and mesenchymal origin(1). Rarely, it may arise from the minor salivary glands localized in the hard palate and other parts of oral mucosa (2).
These tumours usually do not recur after adequate surgical excision. Most recurrences can be attributable to inadequate surgical techniques such as simple enucleation leaving behind microscopic pseudopod-like extensions (2).

This has not been reported in Sri Lanka previously.

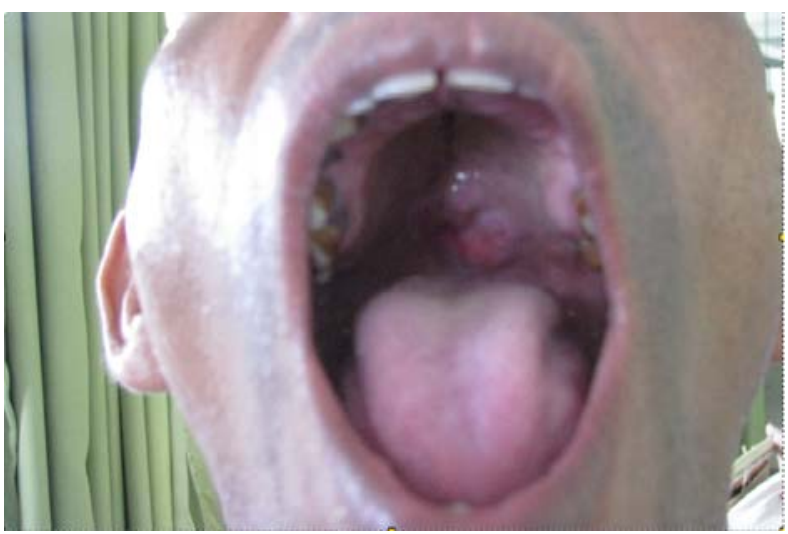

Figure 1

\section{References}

1. Suen JY, Synderman NL. Benign neoplasms of the salivary glands. In: Cummings CW, Fredrickson JM, Harker LA, Krause CJ, Schuller DE eds. Otolaryngology - Head and Neck Surgery. Mosby Year Book, 2nd edition, Vol. 2, 1993; 1029-1042.

2. Feinmesser R, Gay I. Pleomorphic adenoma of the hard palate: an invasive tumour? Journal of Laryngology and Otology 1983; 97: 1169-71. 